PROCEEDINGS OF THE

AMERICAN MATHEMATICAL SOCIETY

Volume 139, Number 10, October 2011, Pages 3763-3768

S 0002-9939(2011)10763-9

Article electronically published on February 17, 2011

\title{
MAPPING SPACES BETWEEN MANIFOLDS AND THE EVALUATION MAP
}

\author{
YVES FELIX
}

(Communicated by Brooke Shipley)

\begin{abstract}
Let $f: M \rightarrow N$ be a map between simply connected $n$-dimensional manifolds. We suppose that $\operatorname{deg} f \neq 0$. Then the injection of aut $t_{1}(N)$ into the component $\operatorname{Map}(M, N ; f)$ of the mapping space containing $f$ induces an injection on the rational homotopy groups, and the evaluation at the base point $\operatorname{map}(M, N ; f) \rightarrow N$ is zero on the rational homotopy groups of even dimension.
\end{abstract}

This paper concerns a generalization of a theorem of Dan Gotllieb [5] motivated by a question of Dusa McDuff [12]. Let $f: M \rightarrow N$ be a map of closed oriented connected manifolds of the same dimension $n$. We suppose that $M$ and $N$ are pointed and that $f$ preserves the base point. We consider the mapping $\operatorname{space} \operatorname{Map}(M, N ; f)$ of continuous maps homotopic to $f$, and we denote by $\omega: \operatorname{Map}(M, N ; f) \rightarrow N$ the evaluation map at the base point $* \in M, \omega(g)=g(*)$. In [5] Gottlieb proves that if $\pi_{1}(\omega): \pi_{1}(\operatorname{Map}(M, N ; f)) \rightarrow \pi_{1}(N)$ is non-zero, then either the degree of $f$, $\operatorname{deg} f=0$, or the Euler characteristic $\chi(N)=0$.

In this paper we consider what happens with the higher order homotopy groups when $M$ and $N$ are simply connected. Recall first that in the case $f=i d_{N}$, the space $\operatorname{Map}(M, N ; f)$ is the monoid $\operatorname{aut}_{1}(N)$ of self-equivalences of $N$ homotopic to the identity. Then denote the evaluation map by $e v:$ aut $_{1} N \rightarrow N$. The image of $\pi_{q}(e v)$ is called the $q^{t h}$ Gottlieb group of $N, G_{q}(N)$. The groups $G_{q}(N) \otimes \mathbb{Q}$ and the map $e v$ have been intensively studied in rational homotopy. In particular, for each integer $q, G_{2 q}(N) \otimes \mathbb{Q}=0$ and $\sum_{q} \operatorname{dim} G_{q}(N) \otimes \mathbb{Q}<n[2$. On the other hand, by a result of $\mathrm{G}$. Lupton and the author, $\widetilde{H}_{*}(e v ; \mathbb{Q})=0$ if $\chi(N) \neq 0[4$. See also [10, 11, 6] for more recent results on Gottlieb groups.

Since the composition with $f$ induces a continuous map $j_{N}: \operatorname{aut}_{1} N \rightarrow$ $\operatorname{Map}(M, N ; f)$ satisfying $\omega \circ j_{N}=e v$, we have the inclusion $G_{*}(N) \subset \operatorname{Im} \pi_{*}(\omega)$. Our main theorem is essentially a converse to this result.

Theorem 1. (1) If $\operatorname{deg} f \neq 0$, then $\operatorname{Im} \pi_{*}(\omega) \otimes \mathbb{Q}=G_{*}(N) \otimes \mathbb{Q}$.

(2) If $\operatorname{deg} f \neq 0$ and $\chi(N) \neq 0$, then the composition of $\pi_{*}(\omega)$ with the Hurewicz $\operatorname{maphur}_{N}: \pi_{*}(N) \otimes \mathbb{Q} \rightarrow H_{*}(N ; Q)$,

$$
\pi_{*}(\operatorname{Map}(M, N ; f)) \otimes \mathbb{Q} \rightarrow H_{*}(N ; \mathbb{Q}),
$$

is zero.

Received by the editors July 17, 2010 and, in revised form, August 16, 2010 and August 24, 2010.

2010 Mathematics Subject Classification. Primary 55P62; Secondary 55Q52.

(C)2011 American Mathematical Society 
An important step in the proof of Theorem 1 is given by Theorem 2 .

Theorem 2. Suppose deg $f \neq 0$. Then the injection $j_{N}: \operatorname{aut}_{1}(N) \rightarrow \operatorname{Map}(M, N ; f)$ induces an injection on the rational homotopy groups,

$$
\pi_{*}\left(j_{N}\right) \otimes \mathbb{Q}: \pi_{*}\left(\operatorname{aut}_{1}(N)\right) \otimes \mathbb{Q} \rightarrow \pi_{*}(\operatorname{Map}(M, N ; f)) \otimes \mathbb{Q} .
$$

Moreover $\pi_{*}\left(j_{N}\right) \otimes \mathbb{Q}$ admits a retraction $\sigma$ satisfying $\left(\pi_{*}(e v) \otimes \mathbb{Q}\right) \circ \sigma=\pi_{*}(\omega) \otimes \mathbb{Q}$.

Now recall that a continuous map $g: X \rightarrow Y$ is called a rational Gottlieb map if $\pi_{*}(g) \otimes \mathbb{Q}$ maps $G_{*}(X) \otimes \mathbb{Q}$ into $G_{*}(Y) \otimes \mathbb{Q}[13$. For instance, when $X$ is an odddimensional sphere $S^{q}$, then $g$ is a rational Gottlieb map if and only if $[g]$ belongs to the Gottlieb group $G_{*}(Y) \otimes \mathbb{Q}$. We prove

Theorem 3. With the above notation, if $\operatorname{deg} f \neq 0$, then $f$ is a rational Gottlieb map.

For the proofs, we use the machinery of rational homotopy theory as described for instance in [3]. We use more precisely the Poincaré duality model for manifolds given by Lambrechts and Stanley in [8] and the description of the rational homotopy groups of mapping spaces in terms of derivations given by Lupton and Smith in [9].

\section{A CONVENiEnt MODEL FOR $f$}

A Poincaré duality model for a simply connected Poincaré duality complex $X$ of dimension $n$ is a commutative differential graded algebra $(A, d)$ quasi-isomorphic to the Sullivan minimal model of $X$ and satisfying Poincaré duality in dimension $n$. This means that there exists a linear map $\varepsilon: A^{n} \rightarrow \mathbb{Q}$ such that $\varepsilon\left(d A^{n-1}\right)=0$ and such that the induced bilinear forms

$$
A^{k} \otimes A^{n-k} \rightarrow \mathbb{Q}, \quad a \otimes b \mapsto \varepsilon(a b)
$$

are non-degenerate. Such a map $\varepsilon$ is then called an orientation.

Theorem 4 (Lambrechts, Stanley [8]). (1) If $(A, d)$ is a commutative differential graded algebra whose cohomology is a simply connected Poincaré duality algebra, then $(A, d)$ is weakly equivalent to a commutative differential graded algebra $\left(A^{\prime}, d\right)$ that is a simply connected Poincaré duality algebra.

(2) Moreover, if $A$ is finite type, $A^{0}=\mathbb{Q}, A^{1}=0, A^{2} \subset K e r d$, and $n \geq 7$, then there is a quasi-isomorphism $\varphi:(A, d) \rightarrow\left(A^{\prime}, d\right)$.

We deduce the following generalization,

Proposition 1. Let $f: M \rightarrow N$ be a map between simply connected $n$-dimensional manifolds, $n \geq 7$. Suppose that $H^{2}(f)$ is injective. Then $f$ admits a Sullivan model of the form

$$
\varphi:(A, d) \rightarrow(B, d)
$$

where $(A, d)$ and $(B, d)$ are Poincaré duality algebras of dimension $n$.

Proof. Let $j:(\bigwedge V, d) \rightarrow(\bigwedge V \otimes \bigwedge W, D)$ be a relative minimal model for $f$ and let $(\bigwedge V, d) \rightarrow(A, d)$ be a quasi-isomorphism with $(A, d)$ a Poincaré duality model for $N$. By taking the tensor product $(A, d) \otimes(\wedge V, d)-$, we get another model of $f$,

$$
1 \otimes j:(A, d) \rightarrow\left(A \otimes \bigwedge W, D^{\prime}\right):=(A, d) \otimes_{(\bigwedge V, d)}(\bigwedge V \otimes \bigwedge W, D) .
$$

Since $H^{2}(f)$ is injective, $(A \otimes \bigwedge W)^{1}=0$. By Theorem 4(2) there then exists a quasi-isomorphism $g:\left(A \otimes \bigwedge W, D^{\prime}\right) \rightarrow(B, d)$ where $(B, d)$ is a Poincaré duality model of $M$. We define $\varphi:(A, d) \rightarrow(B, d)$ by $\varphi=g \circ(1 \otimes j)$. 
Our next proposition explains the structure of the model $\varphi$ when $\operatorname{deg} f \neq 0$.

Proposition 2. With the above notation, if $\operatorname{deg} f \neq 0$, then

(1) the map $\varphi$ is injective,

(2) the graded vector space $B$ can be decomposed as $B=\varphi(A) \oplus Z$, where $d(Z) \subset Z$ and $Z \cdot \varphi(A) \subset Z$.

Proof. Denote by $\omega$ and $\omega^{\prime}$ rational fundamental classes of $A$ and $B$ with $\varphi(\omega)=\omega^{\prime}$, and denote by $\varepsilon^{\prime}$ the associated orientation of $B$. In particular, $\varepsilon(a)=\varepsilon^{\prime}(\varphi(a))$ for $a \in A$. If $\varphi(a)=0$ for some $a$, then $\varphi(\omega)=0$ because there is an element $a^{\prime}$ such that $a a^{\prime}=\omega$, and so $\varphi$ is injective.

Denote

$$
Z=\left\{x \in B \mid \varepsilon^{\prime}(x \cdot \varphi(A))=0\right\} .
$$

Choose a homogeneous basis $h_{1}, \ldots, h_{m}$ of $A$. Using the Poincaré duality of $A$, there is another family $\left\{h_{j}^{*}\right\} \subset A$ such that $\varepsilon\left(h_{j}^{*} \cdot h_{i}\right)=\delta_{i j}$, where $\delta_{i j}$ is the Kronecker symbol. Then $\varphi\left(h_{1}\right), \ldots, \varphi\left(h_{m}\right)$ and $\varphi\left(h_{1}^{*}\right), \ldots, \varphi\left(h_{m}^{*}\right)$ are homogeneous bases of $\varphi(A)$. If $b \in B$, the element

$$
b^{\prime}=b-\sum_{j} \varepsilon^{\prime}\left(b \cdot \varphi\left(h_{j}\right)\right) \varphi\left(h_{j}^{*}\right)
$$

is in $Z$. This shows that $B=\varphi(A) \oplus Z$. Now for an element $z \in Z$,

$$
\varepsilon^{\prime}(d z \cdot \varphi(a))=\varepsilon^{\prime}(d(z \varphi(a)))-(-1)^{|z|} \varepsilon^{\prime}(z \cdot d \varphi(a))=0 .
$$

This shows that $d(Z) \subset Z$. We also have $Z \cdot \varphi(A) \subset Z$ because $\varepsilon^{\prime}[(z \cdot \varphi(a)) \cdot \varphi(A)]=$ 0 .

\section{MOdel FOR MAPPING SPACES}

In this section we recall the derivation model for the rational homotopy groups of a mapping space $\operatorname{Map}(X, Y ; f)$, where $X$ and $Y$ are simply connected CW complexes and $\operatorname{dim} X<\infty$. Recall that under those hypothesis, $\operatorname{Map}(M, N ; f)$ is a nilpotent space whose rationalization is obtained by taking the composition with a rationalization of $N, \ell: N \rightarrow N_{0}$ ([7]).

Let $\ell:(\bigwedge V, d) \rightarrow(A, d)$ be a model for $f$, with $(\bigwedge V, d)$ a Sullivan (nonnecessarily minimal) model for $Y$ and $(A, d)$ a connected model for $X$. We consider the differential graded vector space $\left(\operatorname{der}^{\ell}(\bigwedge V, A), \delta\right)$ of $\ell$-derivations, where $\operatorname{der}^{\ell}(\bigwedge V, A)_{m}$ is the vector space of linear maps of degree $m, \theta:(\bigwedge V)^{*} \rightarrow A^{*-m}$ for which $\theta(x y)=\theta(x) \ell(y)+(-1)^{m|x|} \ell(x) \theta(y)$. The differential $\delta$ is defined as usual by

$$
\delta \theta=d \circ \theta+(-1)^{m+1} \theta \circ d .
$$

From now on, we restrict to $\operatorname{Der}^{\ell}(\bigwedge V, A)$, the positive $\ell$-derivations,

$$
\operatorname{Der}^{\ell}(\bigwedge V, A)_{r}= \begin{cases}\operatorname{der}^{\ell}(\bigwedge V, A)_{r}, & \text { if } r>1, \\ Z \operatorname{der}^{\ell}(\bigwedge V, A)_{1}, & \text { if } r=1,\end{cases}
$$

where $Z$ denotes the space of cycles.

It is well known that if $g:(\bigwedge W, d) \rightarrow(\bigwedge V, d)$ is a quasi-isomorphism, then the induced map $\operatorname{Der}^{\ell}(\bigwedge V, A) \rightarrow \operatorname{Der}^{\ell \circ g}(\bigwedge W, A)$ is a quasi-isomorphism. In the same way, if $h:(A, d) \rightarrow\left(A^{\prime}, d\right)$ is a quasi-isomorphism, then the induced map $\operatorname{Der}^{\ell}(\bigwedge V, A) \rightarrow \operatorname{Der}^{h \circ \ell}\left(\bigwedge V, A^{\prime}\right)$ is also a quasi-isomorphism (see for instance [1], 
Theorem 2.8). The following theorem follows then directly from [9, where the theorem is proved when $(\bigwedge V, d)$ is minimal and $(A, d)$ is the minimal model of $X$.

Theorem 5 ([9], [1], Theorem 3.8). With the above notation,

(1) we have natural isomorphisms of graded vector spaces

$$
\begin{gathered}
H_{*}\left(\operatorname{Der}^{\ell}(\bigwedge V, A)\right) \cong \pi_{*}\left(\operatorname{Map}_{*}(X, Y ; f)\right) \otimes \mathbb{Q}, \\
H_{*}\left(\operatorname{Der}^{\ell}\left(\bigwedge V, A^{+}\right)\right) \cong \pi_{*}\left(\operatorname{Map}_{*}(X, Y ; f)\right) \otimes \mathbb{Q},
\end{gathered}
$$

where $\operatorname{Map}_{*}(X, Y ; f)$ denotes the subspace of $\operatorname{Map}(M, N ; f)$ consisting of pointed maps.

(2) Denote by $\alpha: A \rightarrow \mathbb{Q}$ the augmentation. Then the composition with $\alpha$ induces a morphism of complexes that is a model for $\pi_{*}(\omega) \otimes \mathbb{Q}$; i.e., we have a commutative diagram

$$
\begin{array}{ccc}
H_{*}\left(\operatorname{Der}^{\ell}(\bigwedge V, A)\right) & \stackrel{\widehat{\alpha}}{\rightarrow} & H_{*}\left(\operatorname{Der}^{\alpha \circ \ell}(\bigwedge V, \mathbb{Q})\right) \\
\cong \uparrow & \cong H_{*}(\operatorname{Hom}(V, \mathbb{Q})) \\
\pi_{*}(\operatorname{Map}(X, Y ; f)) \otimes \mathbb{Q} & \stackrel{\pi_{*}(\omega)}{\rightarrow} & \pi_{*}(N) \otimes \mathbb{Q} .
\end{array}
$$

\section{Proof of Theorem 2}

We first consider the case $n \geq 7$ and we use the notation of section 1 . In particular, $\varphi:(A, d) \rightarrow(B, d)$ is a model for $f$, and $(A, d)$ and $(B, d)$ are Poincaré duality models for $N$ and $M$. Let $\psi:(\bigwedge V, d) \rightarrow(A, d)$ be a surjective Sullivan model for $(A, d)$, and then let $\rho=\varphi \circ \psi:(\bigwedge V, d) \rightarrow(B, d)$.

Recall that $B=\varphi(A) \oplus Z$ with $d Z \subset Z$ and $Z \cdot \varphi(A) \subset Z$. Then $B=\rho(\bigwedge V) \oplus Z$ with $Z \cdot \rho(\bigwedge V) \subset Z$. Define a linear map

$$
\Phi: \operatorname{Der}^{\rho}(\bigwedge V, B) \rightarrow \operatorname{Der}^{\psi}(\bigwedge V, A)
$$

by $\Phi(\theta)=p \circ \theta$, where $p: B \rightarrow \varphi(A) \cong A$ is the linear projection with kernel $Z$.

Lemma 1. The morphism $p \circ \theta$ is a $\psi$-derivation.

Proof. Write $\theta(a)=x+z$ with $z \in Z$ and $x=\varphi(p \theta(a))$ and $\theta(b)=x^{\prime}+z^{\prime}$ with $z^{\prime} \in Z$ and $x^{\prime}=\varphi(p \theta(b))$. Then,

$$
\begin{aligned}
p \theta(a b) & =p\left(\theta(a) \cdot \rho(b)+(-1)^{|a||\theta|} \rho(a) \cdot \theta(b)\right) \\
& =p\left(x \rho(b)+(-1)^{|\theta| \cdot|a|} \rho(a) x^{\prime}\right) \\
& =p\left(\varphi(p \theta(a) \cdot \psi(b))+(-1)^{|\theta| \cdot|a|} \varphi(\psi(a) \cdot p \theta(b))\right) \\
& =p \theta(a) \cdot \psi(b)+(-1)^{|a||\psi|} \psi(a) \cdot p \theta(b) .
\end{aligned}
$$

Lemma 2. $\Phi$ is a morphism of complexes.

Proof.

$$
\delta(p \theta)=d p \theta-(-1)^{|\theta|}(p \theta) d=p(d \theta)-(-1)^{|\theta|} p(\theta d)=p(\delta \theta) .
$$

Since $A$ is a subalgebra of $B$, the injection of $A$ into $B$ induces a morphism of complexes

$$
\Psi: \operatorname{Der}^{\psi}(\bigwedge V, A) \rightarrow \operatorname{Der}^{\rho}(\bigwedge V, B)
$$

such that $\Phi \circ \Psi=i d \operatorname{Der}^{\psi}(\wedge V, A)$. 
Proof of Theorem 2 when $n \geq 7$. When $n \geq 7$, Theorem 5 directly yields that the induced injection $\Psi: \operatorname{Der}^{\psi}(\bigwedge V, A) \rightarrow \operatorname{Der}^{\rho}(\bigwedge V, B)$ induces in homology the morphism $\pi_{*}\left(j_{N}\right) \otimes \mathbb{Q}$. A retraction $\sigma$ to $\pi_{*}\left(j_{N}\right) \otimes \mathbb{Q}$ is given by $H_{*}(\Phi)$.

Now the commutativity of the diagram

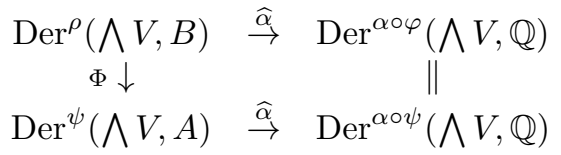

implies in homology that $\left(\pi_{*}(e v) \otimes \mathbb{Q}\right) \circ \sigma=\pi_{*}(\omega) \otimes \mathbb{Q}$.

Proof of Theorem 2 for $n \leq 6$. Let define $g=f \times i d_{S^{6}}: M \times S^{6} \rightarrow N \times S^{6}$. If $f^{\prime} \sim f$, then $f^{\prime} \times i d \sim f \times i d$. This defines a continuous map

$$
e: \operatorname{Map}(M, N ; f) \rightarrow \operatorname{Map}\left(M \times S^{6}, N \times S^{6} ; f \times i d\right) .
$$

On the other hand, if $g^{\prime}: M \times S^{6} \rightarrow N \times S^{6}$ is homotopic to $f \times i d$, then we consider the composition

$$
M \stackrel{h}{\longrightarrow} M \times S^{6} \stackrel{g^{\prime}}{\longrightarrow} N \times S^{6} \stackrel{p}{\longrightarrow} N
$$

where $* \in S^{6}$ is the base point, $h(m)=(m, *)$ and $p(r, x)=r$. Since $p g^{\prime} h \sim f$, this gives a continuous map

$$
r: \operatorname{Map}\left(M \times S^{6}, N \times S^{6} ; f \times i d\right) \rightarrow \operatorname{Map}(M, N ; f) .
$$

Clearly $r \circ e=i d$; i.e., $\operatorname{Map}(M, N ; f)$ is a retract of $\operatorname{Map}\left(M \times S^{6}, N \times S^{6} ; f \times i d\right)$.

Since $\operatorname{dim}\left(N \times S^{6}\right) \geq 7$, we have a map $\sigma$ making commutative the diagram

$$
\begin{array}{ccc}
\pi_{*}(\operatorname{Map}(M, N ; f)) \otimes \mathbb{Q} & \stackrel{\pi_{*}(\omega)}{\longrightarrow} & \pi_{*}(N) \otimes \mathbb{Q} \\
\pi_{*}(e) \downarrow & & \downarrow \pi_{*}\left(h^{\prime}\right) \\
\pi_{*}\left(\operatorname{Map}\left(M \times S^{6}, N \times S^{6}, f \times i d\right)\right) \otimes \mathbb{Q} & \stackrel{\pi_{*}(\omega)}{\longrightarrow} & \pi_{*}\left(N \times S^{6}\right) \otimes \mathbb{Q} \\
\sigma \downarrow & & \| \\
\pi_{*}\left(\text { aut }_{1}\left(N \times S^{6}\right)\right) \otimes \mathbb{Q} & \stackrel{\pi_{*}(e v)}{\longrightarrow} & \pi_{*}\left(N \times S^{6}\right) \otimes \mathbb{Q},
\end{array}
$$

where $h^{\prime}(n)=(n, *)$.

In the same way, $\operatorname{aut}_{1}(N)$ is a retract of $a u t_{1}\left(N \times S^{6}\right)$. Denote by $i: a_{u} t_{1}(N) \rightarrow$ $\operatorname{aut}_{1}\left(N \times S^{6}\right)$ the natural injection. The retraction $\tau$ associates to a map $h$ : $N \times S^{6} \rightarrow N \times S^{6}$ the composition

$$
N \stackrel{h^{\prime}}{\rightarrow} N \times S^{6} \stackrel{h}{\rightarrow} N \times S^{6} \stackrel{p}{\rightarrow} N .
$$

By construction we have $e v \circ \tau=p \circ e v$ and a commutative diagram

$$
\begin{array}{ccc}
\operatorname{aut}_{1}(N) & \stackrel{i}{\rightarrow} & \text { aut }_{1}\left(N \times S^{6}\right) \\
j_{N} \downarrow & & \downarrow j_{N \times S^{6}} \\
\operatorname{Map}(M, N ; f) & \stackrel{e}{\rightarrow} & \operatorname{Map}\left(M \times S^{6}, N \times S^{6}, f \times i d\right) .
\end{array}
$$

Then $\sigma^{\prime}=\left(\pi_{*}(\tau) \otimes \mathbb{Q}\right) \circ \sigma \circ\left(\pi_{*}(e) \otimes \mathbb{Q}\right)$,

$$
\begin{aligned}
\pi_{*}(\operatorname{Map}(M, N ; f)) \otimes \mathbb{Q} & \rightarrow \pi_{*}\left(\operatorname{Map}\left(M \times S^{6}, N \times S^{6} ; f \times i d\right)\right) \otimes \mathbb{Q} \\
& \rightarrow \pi_{*}\left(\text { aut }_{1}\left(N \times S^{6}\right)\right) \otimes \mathbb{Q} \rightarrow \pi_{*}\left(\text { aut }_{1}(N)\right) \otimes \mathbb{Q}
\end{aligned}
$$

is a retraction of $\pi_{*}\left(j_{N}\right) \otimes \mathbb{Q}$, and by construction $\left(\pi_{*}(e v) \otimes \mathbb{Q}\right) \circ \sigma^{\prime}=\pi_{*}(\omega) \otimes \mathbb{Q}$.

Proof of Theorem 1 . Theorem 2 shows that $\operatorname{Im} \pi_{*}(\omega) \otimes \mathbb{Q} \subset \mathrm{G}_{*}(\mathrm{~N}) \otimes \mathbb{Q}$. This proves Theorem 1(1). If $\chi(N) \neq 0, \widetilde{H}_{*}(e v ; \mathbb{Q})=0$, and so by Theorem $2, \operatorname{hur}_{N} \circ\left(\pi_{*}(\omega) \otimes\right.$ $\mathbb{Q})=h u r_{N} \circ\left(\pi_{*}(e v) \otimes \mathbb{Q}\right) \circ \sigma=\widetilde{H}_{*}(e v ; \mathbb{Q}) \circ h u r_{a u t_{1}(N)} \circ \sigma=0$. 
Proof of Theorem 3. Denote by $i_{M}: \operatorname{aut}_{1} M \rightarrow \operatorname{Map}(M, N ; f)$ the map associated to the composition with $f$. The commutativity of the diagram $(q \geq 1)$

$$
\begin{array}{ccccc}
\pi_{q}\left(\text { aut }_{1}(M)\right) \otimes \mathbb{Q} & \stackrel{\pi_{q}\left(i_{M}\right)}{\rightarrow} & \pi_{q}(\operatorname{Map}(M, N ; f) \otimes \mathbb{Q} & \stackrel{\sigma}{\rightarrow} & \pi_{q}\left(\text { aut }_{1}(N)\right) \otimes \mathbb{Q} \\
\pi_{q}(e v) \downarrow & & \downarrow \pi_{q}(e v) & & \downarrow \pi_{q}(e v) \\
\pi_{q}(M) \otimes \mathbb{Q} & \stackrel{\pi_{q}(f)}{\longrightarrow} & \pi_{q}(N) \otimes \mathbb{Q} & = & \pi_{q}(N) \otimes \mathbb{Q}
\end{array}
$$

shows that $f$ maps $G_{*}(M) \otimes \mathbb{Q}$ into $G_{*}(N) \otimes \mathbb{Q}$.

\section{ACKNOWLEDGMENT}

The author would like to thank the referee for helpful comments and suggestions.

\section{REFERENCES}

[1] J. Block and A. Lazarev, André-Quillen cohomology and rational homotopy of function spaces, Advances in Math. 193 (2005), 18-39. MR2132759 (2006a:55014)

[2] Y. Félix and S. Halperin, Rational LS category and its applications, Trans. Amer. Math. Soc. 273 (1982), 1-38. MR664027(84h:55011)

[3] Y. Félix, S. Halperin and J.-C. Thomas, Rational homotopy theory, Graduate Texts in Mathematics, vol. 205, Springer-Verlag, 2001. MR.1802847 (2002d:55014)

[4] Y. Félix and G. Lupton, Evaluation maps in rational homotopy, Topology 46 (2007), 493-506. MR2337558 (2008e:55014)

[5] D.H. Gottlieb, Self coincidence numbers and the fundamental group, J. Fixed Point Theory Appl. 2 (2007), 73-83. MR2336500 (2008h:55002)

[6] Y. Hirato, K. Kuribayashi and N. Oda, A function space model approach to the rational evaluation subgroups, Math. Z. 258 (2008), 521-555. MR.2369043 (2008j:55012)

[7] P. Hilton, G. Mislin and J. Roitberg, Localization of nilpotent groups and spaces, NorthHolland Mathematics Studies, No. 15, North-Holland Publishing Co., 1975. MR0478146 $(57: 17635)$

[8] P. Lambrechts and D. Stanley, Poincaré duality and commutative differential graded algebras, Ann. Scient. Ec. Norm. Sup. 41 (2008), 495-509. MR2489632 (2009k:55022)

[9] G. Lupton and S.B. Smith, Rationalized evaluation subgroups of a map. I. Sullivan models, derivations and $G$-sequences, J. Pure Appl. Algebra 209 (2007), 159-171. MR 2292124 (2008c:55017)

[10] G. Lupton and S.B. Smith, Rationalized evaluation subgroups of a map. II. Quillen models and adjoint maps, J. Pure Appl. Algebra 209 (2007), 173-188. MR.2292125 (2008c:55018)

[11] G. Lupton and S.B. Smith, The evaluation subgroup of a fibre inclusion, Topology Appl. 154 (2007), 1107-1118. MR2298626(2008c:55023)

[12] D. McDuff, The symplectomorphism group of a blow up, Geom. Dedicata 132 (2008), 1-29. MR2396906 (2009c:57044)

[13] T. Yamaguchi, A rational obstruction to be a Gottlieb map, J. Homotopy Relat. Struct. 5 (2010), 97-111. MR2610172

Institut Mathematique, Université Catholique de Louvain, 2, Chemin du Cyclotron, 1348 Louvain-La-Neuve, Belgium 